\title{
Calculation of the Centrally Symmetric and Vortex Forces Acting on Momentum in Distortion Tensor Field: Explanation of High-Temperature Plasma and Vortex Motions
}

\author{
Aleksander Yakovlevich Braginsky \\ Institute of Physics, Southern Federal University, Rostov-on-Don, Russia \\ Email: a.braginsky@mail.ru
}

How to cite this paper: Braginsky, A.Y. (2019) Calculation of the Centrally Symmetric and Vortex Forces Acting on Momentum in Distortion Tensor Field: Explanation of High-Temperature Plasma and Vortex Motions. Journal of Applied Mathematics and Physics, 7, 809-828. https://doi.org/10.4236/jamp.2019.74055

Received: January 7, 2019

Accepted: April 13, 2019

Published: April 16, 2019

Copyright $\odot 2019$ by author(s) and Scientific Research Publishing Inc. This work is licensed under the Creative Commons Attribution International License (CC BY 4.0).

http://creativecommons.org/licenses/by/4.0/

\begin{abstract}
The paper deals with calculation of the centrally symmetric and vortex forces for the momentum of a particle in the distortion tensor field from the action minimum, by analogy with the calculation of forces for a charge in an electromagnetic field. It is demonstrated that: 1) The compensating interaction tensor corresponds to the distortion tensor in a solid. 2) The centrally symmetric force of the distortion tensor acts on the momentum as a charge, and is analogous to the Coulomb force. In a gas, it results in change in the momentum value of the molecules exponentially to some extent. The action of this force explains the high-temperature plasma in the gas. 3) The vortex force of the distortion tensor is equivalent to the Peach-Koehler force in a solid. It acts on the momentum flow, similar to the Lorentz magnetic force, and explains the vortex motions in space, in the form of "black holes", and in the atmosphere, in the form of cyclones and anticyclones.
\end{abstract}

\section{Keywords}

Distortion Tensor, High-Temperature Plasma, Vortex Motions, Turbulence, Gauge Field, Explosion of a Meteorite

\section{Introduction}

This paper deals with calculation of the forces acting on the momentum $p_{i}$ in the field of the distortion tensor $A_{i j}$, by analogy with the calculation of the forces acting on a charge in an electromagnetic field [1]. In order to carry out such calculations, one must first prove that the momentum behaves like a charge in the tensor interaction field $A_{i j}$. 
What kind of momentum is it and what is the tensor interaction field? In [2], a minimal interaction was obtained, where the charge is the wave vector $\kappa_{i}$, and the compensating field is the distortion tensor $A_{i j}$. It is known that the wave vector is proportional to the quantum momentum, so one may put the task of calculating the forces acting on a quantum momentum in the field of the distortion tensor, in analogy with the well-known task of field theory for electrodynamics [1]. But first, let's see what the distortion tensor is and where the minimal interaction comes from.

The distortion tensor $A_{i j}$ was introduced into the continuum theory of dislocations as a tensor, the antisymmetric derivative of which defines the dislocation density field [3]:

$$
\rho_{i j}=-e_{j k n} \partial A_{i n} / \partial X_{k}
$$

and the contour integral is defined by the Burgers vector: $\mathrm{B}_{i}=-\oint_{L} A_{i j} \mathrm{~d} X_{j}$. The distortion tensor $A_{i j}$ is a generalization of the strain tensor and describes plastic deformations.

On the other hand, in [2] it was proved that the distortion tensor $A_{i j}$ is a compensating field for the Landau order parameter (OP) $\psi_{\vec{k}}$, which describes the $A$ phase of helimagnetics $\mathrm{Fe}_{0.5} \mathrm{Co}_{0.5} \mathrm{Si}, \mathrm{MnSi}, \mathrm{FeGe}$ in a magnetic field [4] [5] [6].

The necessity of introducing a compensating interaction tensor $A_{i j}$ for the OP induced by the subgroup of elementary translations $\hat{a}_{q} \psi_{\boldsymbol{k}}=\exp \left(i \delta_{i q} k_{i} a_{q}\right) \psi_{\boldsymbol{k}}$ into the inhomogeneous Landau potential was dictated by the local representation of the $k_{i}=k_{i}\left(X_{j}\right)$ OP, when describing helimagnetics in a magnetic field; here $\delta_{i q}$ is the Kronecker symbol. Since for the dependence $k_{i}=k_{i}\left(X_{j}\right)$ the derivative $\partial \psi_{k} / \partial X_{j}$ is not an eigenfunction for the operator of elementary translations:

$$
\hat{a}_{q} \frac{\partial \psi_{\boldsymbol{k}}}{\partial X_{j}}=\frac{\partial}{\partial X_{j}}\left(\mathrm{e}^{i k_{q} a_{q}} \psi_{\boldsymbol{k}}\right)=\mathrm{e}^{i k_{q} a_{q}}\left[i \frac{\partial\left(k_{q} a_{q}\right)}{\partial X_{j}} \psi_{\boldsymbol{k}}+\frac{\partial \psi_{\boldsymbol{k}}}{\partial X_{j}}\right] .
$$

In order to set up an invariant potential or Lagrangian, it is necessary to extend the derivative:

$$
D_{j} \psi_{\boldsymbol{k}}=\left(\frac{\partial}{\partial X_{j}}-i \sum_{n} \kappa_{n} A_{n j}\right) \psi_{\boldsymbol{k}}
$$

by means of compensating tensor field $A_{i j}$, which is transformed as:

$$
\hat{a}_{q}\left(\kappa_{i} A_{i j}\right)=\kappa_{i} A_{i j}+\delta_{i q} \partial\left(k_{i} a_{q}\right) / \partial X_{j}
$$

The extended derivative (2) is an eigenfunction of the $\hat{a}_{q}$ translation operator. In this case, the interaction charge is the coefficient before the compensating field $A_{i j}$ in the extended derivative. The compensating field $A_{i j}$ is tensor, since the interaction charge $\kappa_{i}$ is vector, and its components are related to the corresponding components of the translation subgroup.

A similar situation of a local representation $k_{i}=k_{i}\left(X_{j}\right)$ for a OP takes place 
in the de Gennes model [7] [8]. De Gennes described the phase transition of nematic-SmA (smectic-A) and deformation in SmA by OP $\psi_{\boldsymbol{k}}$. The vector $\boldsymbol{k}$ in SmA is equal to $\boldsymbol{k}=2 \pi \boldsymbol{n} / \boldsymbol{d}$, where $\boldsymbol{n}$ is the normal vector, and $d$ is the distance between layers in smectic. De Gennes believed that in case of deformations in SmA: $n_{i}=n_{i}\left(X_{j}\right)$, hence: $k_{i}=k_{i}\left(X_{j}\right)$.

The extended derivative (2) defines the minimum interaction between the compensating interaction tensor $A_{i j}$ and the OP components $\psi_{\boldsymbol{k}}$. According to the field theory [9], this means that the field $A_{i j}$ is an independent physical variable analogous to the electromagnetic potential $A_{j}$, and manifests itself in the form of observable antisymmetric derivatives (1) everywhere, and not only in a solid.

The compensating field $A_{i j}(\boldsymbol{X})$ is transformed as a second-rank tensor in the space of macroscopic coordinates $\left\{X_{i}\right\}$, where $\boldsymbol{X}$ characterizes the macroscopic small volume of a continuous medium in the Lifshitz formalism [10]. Therefore, the coordinates here and thereafter will be capitalized.

It is known that the electromagnetic potential $A_{j}$ is transformed as a vector field. The compensating Yang-Mills fields also represent a vector in space $\left\{X_{i}\right\}$, and it is already transformed in the space of internal gauge symmetries. Tensor compensating fields $A_{i j}$ have never been previously studied in field theory. As noted in the introduction [9], the numerous attempts to associate the compensating gauge fields to the space-time symmetry have not been successful.

The tensor compensating field of interaction $A_{i j}$ appeared naturally in the Landau theory in the course of formation of the invariants from OP derivatives with local translational properties $k_{i}=k_{i}\left(X_{j}\right)$ [11]. It is induced by a subgroup of translations, therefore it is tensorial. The essence of paper [11] lied in the fact that the symmetry group is global, and the representation of the translation subgroup is local, which describes the physical state in the form of OP or wave function. This makes it possible not to introduce the local abstract gauge groups into the theory in order to obtain a minimal interaction, but to use local representations of the usual translation subgroup. We show, that in the solid, the compensating field $A_{i j}(\boldsymbol{X})$ (2.3) is equivalent to the distortion tensor (1).

The dependence on the scalar product coordinate $\partial\left(k_{i} a_{q}\right) / \partial X_{j}$ in (3) can be defined both by local representation of the OP: $k_{i}=k_{i}\left(X_{j}\right)$, and local symmetry in the low-symmetric deformed state: $a_{i}=a_{i}\left(X_{j}\right)$. The case of a local representation $k_{i}=k_{i}\left(X_{j}\right)$ and a global subgroup of translations were considered in [2]. This is important in describing the phase transition. But in a low-symmetric state, in case of structural phase transition, here is the situation when the lattice constant depends on the coordinate. Therefore, let us consider the case of a low-symmetric state with local translational symmetry, when $a_{i}=a_{i}\left(X_{j}\right)$ and $k_{i}=$ const .

On the other hand, it follows from the definition of the displacement vector $\boldsymbol{u}(\boldsymbol{X})=\boldsymbol{X}-\boldsymbol{X}_{0}$ that $\hat{a}_{i}(\boldsymbol{u}(\boldsymbol{X}))=\boldsymbol{X}+\boldsymbol{a}_{i}(\boldsymbol{X})-\boldsymbol{X}_{0}-\boldsymbol{a}_{i}\left(\boldsymbol{X}_{0}\right)$. When $a_{i}=a_{i}\left(X_{j}\right)$, the translation transformation to the lattice constant is not inva- 
riant under inhomogeneous deformations: $\hat{a}_{i}\left(\partial u_{i} / \partial X_{j}\right)=\partial u_{i} / \partial X_{j}+\partial a_{i} / \partial X_{j}$. Here it is taken into account that $\partial a_{i}\left(\boldsymbol{X}_{0}\right) / \partial X_{j}=0$ in the undeformed state.

Since the distortion tensor $A_{i j}$ is a generalization of $\partial u_{i} / \partial X_{j}$, then in cases of translations to the lattice constant it is also transformed as $\partial u_{i} / \partial X_{j}$ :

$$
\hat{a}_{q}\left(A_{i j}\right)=A_{i j}+\delta_{i q} \partial a_{q} / \partial X_{j}
$$

Whence it follows that the compensating field in (2) is the distortion tensor, if the charge $\kappa_{i}$ in $(2,3)$ is the wave vector: $\kappa_{i}=k_{i}$. Let us prove this.

As a result of the minimal interaction (2), the strain tensor $\sigma_{i j}=-\partial L / \partial A_{i j}$ conjugate to distortion tensor $A_{i j}$ is proportional to the linear combination in the field $A_{i j}:\left(2 \nabla_{j} \varphi-2 \kappa_{i} A_{i j}\right)\left|\psi_{k}\right|^{2}$, where $\varphi$ is the OP phase, since the distortion tensor $A_{i j}$ enters the Lagrangian $L$ or the Landau nonequilibrium potential only in invariant combinations (2): $D_{j} \psi_{k} D_{j}^{*} \psi_{-k}$.

When the strain tensor $\sigma_{p j}$ is identically zero, for example, outside the sample, then the following relation must be satisfied: $\oint_{L} \nabla_{j} \varphi \mathrm{d} X_{j}=\kappa_{i} \oint_{L} A_{i j} \mathrm{~d} X_{j}$, since the fields are not equal to zero in field theory. The integral at the left is quantized:

$$
\oint_{L} \nabla_{j} \varphi \mathrm{d} X_{j}=2 \pi z
$$

where $z \in Z$ is an integer, since in the course of encirclement the phase of OP or wave function can change in multiples of $2 \pi$. The integral at the right, according to (1), is the dislocation density flow or Burgers vector: $\mathrm{B}_{i}=\int_{S_{L}} \rho_{i j} \mathrm{~d} S_{j}$, and according to the Stokes theorem (1): $\kappa_{i} \oint_{L} A_{i j} \mathrm{~d} X_{j}=-\kappa_{i} \mathrm{~B}_{i}$. Whence we receive the minimum value of the Burgers vector: $B_{i \min }=2 \pi / \kappa_{i}$.

This proof is equivalent to proof of magnetic flux quantization $\Phi=\int_{S_{L}} \boldsymbol{B} \mathrm{d} \boldsymbol{S}$ in Abrikosov vortices in superconductivity [12], which follows from the extended Ginzburg-Landau derivative, where the compensating field is the electromagnetic potential $A_{j}$. As is known, the Burgers vector is proportional to the lattice constant $a_{i}=2 \pi / k_{i}$ or the wavelength of the structural OP, for example in SmA [7]. Whence it follows that the charge in (2) is the wave vector $\kappa_{i}=k_{i}$.

This conclusion also demonstrates that the dislocation density $\rho_{i j}$ does not always correspond to the discrete dislocations in a solid, but only when there is a minimum interaction (2). The field $\rho_{i j}$ (1) in a continuous medium, where there is no minimum interaction (2), is the observable intensity of the compensating tensor field $A_{p j}$, analogous to the magnetic field $B_{j}=e_{j k n} \partial A_{n} / \partial X_{k} \quad$ [13] [14].

The name of dislocation density arose in determining dislocations in a continuous medium (1). It must be recognized that this is an unfortunate name for the force intensity of the tensor interaction field $A_{i j}$ (2), since in general it is not connected with the location of atoms in the lattice. The Burgers vector relate 
to the dislocation of atoms in the lattice-the dislocation density flux in the presence of the minimal interaction (2). The vortex invariant intensity of the tensor interaction field (1) may exist in itself in any medium, like a magnetic field. Therefore, from this point on, by $\rho_{i j}$ (1) we will mean the invariant vortex intensity of the compensating tensor interaction field $A_{i j}(2,3)$, which is related to the dislocation density only in a solid.

Note the fact that sometimes in the reference literature, the distortion tensor is the derivative of the displacement vector with respect to the coordinate: $W_{i j}=\partial u_{i} / \partial X_{j}$. Such a definition has nothing to do with the definition of the distortion tensor (1), since $e_{j k n} \partial W_{i n} / \partial X_{k}=0$. In this paper we are talking about the distortion tensor (1), which is defined as an unobservable compensating interaction field $(2,3)$. It is unobservable because it is invariant for elementary translations (4), in contrast to the invariant antisymmetric derivative (1).

In [13] [14], a Lagrangian for the 4-tensor of distortion was formed $v_{i}, A_{i j}$ :

$$
L=p_{i} v_{i}-\sigma_{i j} A_{i j}+\frac{\gamma}{2}\left(\frac{1}{c^{2}} \varepsilon_{i j} \varepsilon_{i j}-\rho_{i j} \rho_{i j}\right)
$$

and a system of state equations similar to the Maxwell equations is obtained [14]:

$$
\begin{gathered}
p_{i}=-\frac{\gamma}{c^{2}} \frac{\partial \varepsilon_{i j}}{\partial X_{j}} \\
\sigma_{i j}=\gamma e_{j k p} \frac{\partial \rho_{i p}}{\partial X_{k}}-\frac{\gamma}{c^{2}} \frac{\partial \varepsilon_{i j}}{\partial T}
\end{gathered}
$$

where

$$
\varepsilon_{i j}=-\partial v_{i} / \partial X_{j}+\partial A_{i j} / \partial T
$$

the centrally symmetric intensity of 4 -tensor of the distortion $v_{i}, A_{i j}$, and $\rho_{i j}$ (1) is the vortex intensity. Here $v_{i}$ is the speed, or the fourth component of the distortion tensor, $p_{i}$ is the momentum, $\sigma_{i j}$ is the strain tensor, $\gamma$ is the dimension factor, and $c$ is the sound speed.

By differentiating the state Equations (6) and (7), we obtain the continuity equation:

$$
\partial \sigma_{i j} / \partial X_{j}=\partial p_{i} / \partial T
$$

which is a differential expression for the law of conservation of momentum.

Thus, it is little wonder that the wave vector $\kappa_{i}$ is a charge in (2). If we pass on to the dynamic model and write the Lagrangian for the model with an extended derivative (2), then the continuity equations in such a model will represent the differential law of conservation of momentum(9). Since the distortion tensor $A_{i j}$ is conjugate to the strain tensor $\sigma_{i j}$, and the speed $v_{i}$ is conjugate to the momentum $p_{i}$ (5). After multiplying the extended derivative (2) by the Planck constant $\hbar$, it becomes obvious that the interaction charge is a quantum momentum $\hbar \kappa_{i}=p_{i}$, which was foreseeable, since the law of conser- 
vation of momentum is associated with translation symmetry (9).

For the first time, the assumption that the distortion tensor $A_{i j}$ and the density of dislocations $\rho_{i j}$ (1) are interaction fields was made by Kadić and Edelen [13]. They constructed a so-called gauge theory of dislocations from the analogy with electrodynamics. However, the Kadić-Edelen theory did not contain a minimal interaction, since the linear translationally invariant combination with the displacement vector derivative (4): $D_{j} u_{i}=\partial u_{i} / \partial X_{j}+A_{i j}^{\prime}$ is not an extended derivative and does not contain a charge of interaction (2). The Kadić-Edelen distortion tensor $A_{i j}^{\prime}$ is equal to the usual distortion tensor $A_{i j}$ with a minus sign: $A_{i j}^{\prime}=-A_{i j}$. Please note that the change in the signature does not impact the Kadić-Edelen state Equation (6.7), but only the definitions of their intensities (1.8), which are given here in accordance with [3].

On the other hand, an extended derivative was constructed in [2] and interaction (2) is given from the translational invariance of the inhomogeneous Landau potential. Consequently, the compensating field of the distortion tensor $A_{i j}$ is indeed an independent interaction field, analogous to the electromagnetic potential $A_{j}$. But it does not complement the derivative of the displacement vector, as Kadić-Edelen thought in [13], but generalizes it according to the definitions (1) [3] and (2) [2].

Moreover, by means of the minimal interaction (2), it was possible to show that the anti-symmetric derivative of the tensor field $\rho_{i j}(1)$ is really connected with discrete dislocations in a solid, since the minimal interaction (2) results in the quantization of the Burgers vector. In the Kadić-Edelen theory, it is impossible to connect the dislocation density field $\rho_{i j}$ with dislocations in a solid, since there is no minimal interaction therein and there is no interaction charge-wave vector $\kappa_{i}(2)$.

For the first time, Kröner [15] noted the analogy between the continuum theory of stationary dislocations and magnetostatics. Since in static $\partial \sigma_{i j} / \partial X_{j}=0$, the strain tensor could be represented as anti-symmetric derivative of the tensor potential $\Lambda_{i j}$, which was called the Kröner potential: $\sigma_{i j}=e_{j k n} \partial \Lambda_{i n} / \partial X_{k}$. Based on logical considerations, Kröner believed that the current density $j_{i}$ is similar to the dislocation density $\rho_{i j}$, and the magnetic induction $B_{j}$ is analogous to the strain tensor $\sigma_{i j}$. However, the fields $B_{j}$ and $\rho_{i j}$ are vortex fields and are determined in connection with the gradient invariance, and the vortex expressions for $j_{i}, \sigma_{i j}$ are a consequence of magnetostatics and stationary equilibrium. In dynamics, the current density $j_{i}$ and the strain tensor $\sigma_{i j}$ are not vortex fields. Therefore, in the general case, the vortex density of dislocations $\rho_{i j}$ cannot be analogous to the current density $j_{i}$, nor in the stationary particular case.

In the initial interpretation of physical fields in [11], it was erroneously assumed that the dislocation density field $\rho_{i j}$ cannot be an interaction field analogous to the magnetic field $B_{j}$, since there are no dislocations in the air. An erroneous analogy in the interpretation of physical values [11] [16], based on the ideas of Kröner and Kosevich [15] [17], did not make it possible earlier to obtain 
the results presented here based on the minimal interaction (2), obtained in 1990 from translational symmetry.

In [2], as illustrated by the two-dimensional model, it was proved that the distortion tensor $A_{i j}$ is a compensating interaction field (2), and the wave vector $\kappa_{i}$ is the charge of such interaction.

In the dynamic model, the continuity equations associated with the translational invariance of the Lagrangian in case elementary translations are a differential representation of the law of conservation of momentum (9). Whence it follows, that the momentum is the charge of the minimal interaction induced by the subgroup of translations.

Herein before, it was proved by direct calculations that the distortion tensor $A_{i j}$ in a solid is a compensating field for the local representation of the translation subgroup and that the quantum momentum $\hbar \kappa_{i}=p_{i}$ is a charge in the extended derivative (2).

Kröner and Kadić-Edelen built their theories based on analogy with magnetostatics and electrodynamics, respectively, and therefore nothing could be proved from analogy. The interaction (2) was obtained from translational symmetry, which is connected with the law of conservation of momentum. A quantum momentum or a wave vector is the charge of the interaction (2) and determines the physical fields. Therefore, the model (2) has no and cannot have any dualism in the definition of physical values. The symmetry group defines the interaction and this group is a subgroup of translations. The quantum momentum is the charge of the interaction (2), and is a result, it determines the interaction fields $(6,7)$ and their intensities $(1,8)$. Thus, the local representation of the translation sub-group $k_{i}=k_{i}\left(X_{j}\right)$ fixes the physical model and the minimal interaction (2).

In this paper, the compensating field $A_{i j}(2,3)$ will be called the tensor interaction field, since there is no tensor interaction field in gauge field theory [9], and it has never been previously investigated. The antisymmetric derivative of the interaction tensor (1), which is the density of dislocations in a solid, will be called the vortex intensity of the interaction tensor. The vortex intensity $\rho_{i j}$ (1) corresponds to the magnetic field in electrodynamics $B_{j}=e_{j k n} \partial A_{n} / \partial X_{k}$. In the general case, it is not connected with dislocations and is not always quantized. For example, $\rho_{i j}$ is not quantized in a continuous medium, which has no minimum interaction (2). In this case, the continuous medium can be either a solid, or a gas or a liquid.

As in the Kadić-Edelen theory, we complete a definition of the tensor $A_{i j}$ and introduce the fourth component of the compensating tensor field $A_{i j}$ in the form of a velocity field $v_{i}$, which is conjugate to momentum $p_{i}$ as a charge: $p_{i}=\partial L / \partial v_{i} \quad$ (5). The velocity field is converted as:

$\hat{a}_{q}\left(\kappa_{i} v_{i}\right)=\kappa_{i} v_{i}+\delta_{i q} \partial\left(k_{i} a_{q}\right) / \partial T$, and compensates for

$D_{0} \psi_{k}=\left(\frac{\partial}{\partial T}-i \sum_{n} \kappa_{n} v_{n}\right) \psi_{\vec{k}}$ time derivatives of the OP. In a solid, the field $v_{i}$ 
is a generalization of the derivative $\partial u_{i} / \partial T$.

The purpose of this paper is to calculate the forces acting on the momentum in the field of the 4-tensor of interaction $v_{i}, A_{i j}$. We will find these forces from the minimum action, by analogy with the calculation of the Coulomb and Lorentz forces for electric charge in an electromagnetic field. For this purpose, it is sufficient to know that the 4-tensor $v_{i}, A_{i j}$ is an independent interaction field and that its charge is a quantum momentum or wave vector $\kappa_{i}=p_{i} / \hbar$

\section{Calculation of the Forces Acting on the Momentum in the Distortion Tensor Field from the Action Minimum}

The action describing the charge motion in an electromagnetic field $\varphi, A_{j}$, [1], has the form: $S_{e l}=-\frac{e}{c^{\prime}} \int_{a}^{b}\left(-A_{j} \mathrm{~d} X_{j}+\varphi \mathrm{d} T\right)$, where $e$ is the electric charge, $c^{\prime}$ is the velocity of light. Taking into account the space-time signature, the action for the wave vector $\kappa_{p}$ (2) in the field of the 4-tensor $v_{i}, A_{i j}$ interaction, has the form:

$$
S_{s t}=-\hbar \kappa_{i} \int_{a}^{b}\left(A_{i j} \mathrm{~d} X_{j}+v_{i} \mathrm{~d} T\right)
$$

Here $\hbar$ is the dimension factor. It is evident, that this is Planck's constant, since the action has the physical dimension the same as Planck's constant. The fact that the coefficient $\hbar$ is the Planck's constant follows from the continuity equations, which for the model (2) have the form of a differential representation of the law of conservation of momentum (9), therefore we can write it as $\hbar \kappa_{i}=p_{i}$. However, we leave this factor in (10) and verify after calculations that the factor $\hbar$ is really Planck's constant.

The Lagrangian of action (10) has the form:

$$
L_{s t}=-\hbar \kappa_{i} A_{i j} v_{j}-\hbar \kappa_{i} v_{i}
$$

where $v_{j}=\mathrm{d} X_{j} / \mathrm{d} T$. Generalized momentum $\mathrm{d} L / \mathrm{d} v_{j}$ [1], for Lagrangian $L=L_{0}+L_{s t}$, taking into account 4-tensor $v_{i}, A_{i j}$ (11), will be:

$$
\mathrm{d} L / \mathrm{d} v_{j}=P_{j}-\hbar \kappa_{i} A_{i j}
$$

here $P_{j}$ is common momentum, equal to $\mathrm{d} L_{0} / \mathrm{d} v_{j}=P_{j} \quad$ [1].

According to $(11,12)$, from Euler-Lagrange equations $\frac{\mathrm{d}}{\mathrm{d} T} \frac{\partial L}{\partial v_{j}}=\frac{\partial L}{\partial X_{j}}$ for particle in interaction tensor field we receive:

$$
\frac{\mathrm{d} P_{j}}{\mathrm{~d} T}-\hbar \kappa_{i} \frac{\mathrm{d} A_{i j}}{\mathrm{~d} T}=-\hbar \kappa_{i} \frac{\partial}{\partial X_{j}}\left(A_{i j} v_{j}\right)-\hbar \kappa_{i} \frac{\partial v_{i}}{\partial X_{j}}
$$

From where, differentiating by parts the second summand in left part (13): $\frac{\mathrm{d} A_{i j}}{\mathrm{~d} T}=\frac{\partial A_{i j}}{\partial T}+\frac{\partial A_{i j}}{\partial X_{k}} v_{k}$ and transforming the first summand in the right part by the well-known formula from differential geometry:

$$
\frac{\partial}{\partial X_{j}}\left(A_{i j} v_{j}\right)=\frac{\partial A_{i j}}{\partial X_{j}} v_{j}+e_{j m n} e_{n p q} v_{m} \frac{\partial A_{i q}}{\partial X_{p}}
$$


(here we considered that $v_{j}$ and $X_{j}$ are independent variables [1]), we receive:

$$
\frac{\mathrm{d} P_{j}}{\mathrm{~d} T}-\hbar \kappa_{i} \frac{\partial A_{i j}}{\partial T}=-\hbar \kappa_{i} \frac{\partial v_{i}}{\partial X_{j}}-\hbar \kappa_{i} e_{j m n} e_{n p q} v_{m} \frac{\partial A_{i q}}{\partial X_{p}}
$$

Then the expression for the force acting on a particle with a charge $\kappa_{i}$ is as follows:

$$
f_{j}=-\hbar \kappa_{i} \frac{\partial v_{i}}{\partial X_{j}}+\hbar \kappa_{i} \frac{\partial A_{i j}}{\partial T}-\hbar \kappa_{i} e_{j m n} e_{n p q} v_{m} \frac{\partial A_{i q}}{\partial X_{p}}
$$

This force is the sum of two forces: the centrally symmetric and vortex forces of the tensor interaction field. Substituting $\hbar \kappa_{i}=p_{i}$, we obtain:

$$
f_{j}=-p_{i} \frac{\partial v_{i}}{\partial X_{j}}+p_{i} \frac{\partial A_{i j}}{\partial T}-p_{i} e_{j m n} e_{n p q} v_{m} \frac{\partial A_{i q}}{\partial X_{p}}
$$

The centrally symmetric force is the expression:

$$
f_{j}=p_{i} \varepsilon_{i j}=p_{i}\left(-\partial v_{i} / \partial X_{j}+\partial A_{i j} / \partial T\right)
$$

where $\varepsilon_{i j}$ is the centrally symmetric intensity of the tensor interaction field (8), it is analogous to the electric intensity.

In the stationary case, the force (16) has the form of $f_{j}=-p_{i} \partial v_{i} / \partial X_{j}$. In a continuous medium with a density $\rho$, when $p_{i}=\rho v_{i}$, the resulting force results in squared velocity gradient with a minus sign in the Euler equations of hydrodynamics, and squared velocity in the Bernoulli equation [18]. This testifies to the correctness of made calculations with the coefficient $\hbar$ in (10).

The vortex force of the tensor interaction field in $(14,15)$, taking into account expression (1), has the form of:

$$
f_{j}=e_{j m n} p_{i} v_{m} \rho_{\text {in }}
$$

It should be noted, that the centrally symmetric and vortex forces $(16,17)$ could be written at once by analogy with electrodynamics, if we know the invariant intensities of the tensor interaction field $\rho_{i j}$ and $\varepsilon_{i j}(1,8)$, and that the charge is the momentum $(9)$. The forces $(16,17)$ are analogous to the Coulomb force $f_{j}=e E_{j}=e\left(-\partial \varphi / \partial X_{j}-\partial A_{j} / \partial T\right)$ and Lorentz force $f_{j}=e_{j m n} e v_{m} B_{n}$. The difference in signs before the second member of the force (16) and the second member of the Coulomb force is associated with the space-time signature.

Please note that the electromagnetic interaction can be obtained by analogy with $(2,3)$ from the invariance of the Lagrangian with respect to time translations, when the wave function or the $\mathrm{OP}$ is a local representation of the time translation subgroup [16]. In case of such definition, there is no need to postulate a local gauge group in the Lagrangian. Moreover, in this case there is no need to postulate in field theory that the electromagnetic potential changes sign in case of time inversion. The change in the sign of the magnetic field and the electromagnetic potential in case of time inversion follows from the construction 
of extended derivative for the local representation of time translations [16]. Consequently, the electromagnetic potential $\varphi, A_{j}$ may be considered as a time component of the 4-tensor of space-time distortion.

\section{The Vortex Force of the Tensor Interaction Field $A_{i j}$, the Peach-Koehler Force}

We show that the vortex force (17) is the Peach-Koehler force in a solid [3] [19]. The Peach-Koehler force is a force that acts on a dislocation in a solid. For a continuous dislocation distribution, it has the form [13] [14]:

$$
f_{j}=e_{j n m} \rho_{i n} \sigma_{i m}
$$

According to the continuity Equation (9), the momentum flux $p_{i} v_{m}$ in (17), in fact, is the strain tensor with the minus sign: $p_{i} v_{m}=-\sigma_{i m}$. Then the vortex force (17) acting on the momentum in the interaction tensor field $A_{i j}$ can be written in the form: $f_{j}=-e_{j m n} \sigma_{i m} \rho_{i n}$. Using the properties of the anti-symmetric Levi-Civita tensor, we find that the vortex force of the tensor $A_{i j}$ (17), which in the solid is the distortion tensor, is the Peach-Koehler force (18).

However, Pitsch and Keller believed that the intensity acts on a dislocation in a solid. By direct calculations, based on the principle of least action, we proved that the vortex intensity $\rho_{i n}$ of the tensor $A_{i j}$ (1) acts on the momentum flux $p_{i} v_{m}=-\sigma_{i m}$ by the Peach-Koehler force $(17,18)$. The Peach-Koehler force acts on the momentum flux (17) in the same manner as the Lorentz force acts on the charge flow $f_{j}=e_{j m n} e v_{m} B_{n}$.

Expressions for forces (17) and (18) are the same, but the meaning is poles apart. It is not stress field act on the dislocation density (18), as Pitsch and Keller [3] [19] believed, but the dislocation density, as a force field (1), acts on the momentum flux as a charge flow (17), and this momentum is quantum (14).

How does it help? For example, it is known that a large Lorentz force captures electrically charged particles and holds plasma with a temperature of millions of degrees in a tokamak. In this case, the magnetic field is induced by a high current. That's just like the great vortex force (17) in space will capture the momentas of particles and will not let them go back, that explains the phenomenon of "black holes".

Indeed, according to (7), the source of a large field $\rho_{\text {in }}$ is a large pressure, since in gas $\sigma_{i j}=-\delta_{i j} p$. As is known, a large pressure in a gas is generated by a large density fluctuation in a certain volume. Consequently, in the space of large field $\rho_{i n}$ there is a large mass, and, consequently, a large gravitational field. But gravity cannot explain the capture of photons. As is known, the photons do not have mass, and they also do not return from "black holes". However, the photons have a momentum, which is also affected by $\rho_{i n}$ through a vortex force (17), and this quantum momentum is $p_{i}=\hbar \kappa_{i}$. As follows from the calculations $(10,14)$, the vortex force (17) also acts on the quantum momentum. In fact, the phenomenon of "black holes" is an experimental confirmation of the fact that the quantum momentum is a charge for the tensor interaction field $v_{i}$, 
$A_{i j}$ (10).

It was shown above how the vortex force of the tensor field $A_{i j}$ acts in space. The question arises how does the vortex force (17) manifest itself in the atmosphere?

In order to determine the relation between the strain tensor $\sigma_{i j}$ and the tensor $A_{i j}$ in a continuous medium, we use the gauge condition for the Kadić-Edelen 4-tensor $v_{i}, A_{i j}[13][14]$ :

$$
\partial A_{i j} / \partial X_{j}=c^{-2} \partial v_{i} / \partial T
$$

In contrast to the Lorentz gauge condition for the electromagnetic field [1], the condition (19) is called pseudo-Lorentz, this is due to the space-time signature.

Since the distortion tensor (1) was initially determined to within a gradient of the displacement vector: $A_{i j} \rightarrow A_{i j}+\partial u_{i} / \partial X_{j}$, and similarly to $v_{j} \rightarrow v_{j}+\partial u_{j} / \partial T$, then the displacement vector according to (19) satisfies the wave equation: $\partial^{2} u_{i} / \partial X_{j}^{2}=c^{-2} \partial^{2} u_{i} / \partial T^{2}$. This wave equation describes the propagation of mechanical oscillations in a continuous medium with $c$ speed of sound.

It follows from $(9,19)$ and the relation $p_{i}=\rho v_{i}$, which characterizes a continuous medium with a density $\rho$, that $\sigma_{i j}=c^{2} \rho A_{i j}$ in a continuous medium.

It should be noted that here the density $\rho$ and speed of sound $c$ are equilibrium parameters of a continuous medium, they do not depend on local coordinates $X_{i}$, and the 4 -tensor acts as local variables $A_{i j}(\boldsymbol{X}), v_{i}(\boldsymbol{X})$.

In a gas $\sigma_{i j}=-\delta_{i j} p$, therefore in gas $A_{i j}=-\beta \delta_{i j} p$, where $\beta=c^{-2} \rho^{-1}$ is the gas compressibility. Then the vortex intensity $\rho_{i j}$ (1) in the gas is as follows:

$$
\rho_{i j}=\beta e_{j k i} \partial p / \partial X_{k}
$$

We should note, that in gas the vortex intensity is not zero, as one would expect, and the components $\rho_{i j}$ are proportional to the components of the pressure or force gradient. At the same time, the intensity (20) in the gas has no relation to dislocations.

In order to calculate the vortex force (17) in the atmosphere, we use expressions $(17,20)$. Since in a continuous medium the velocity of a continuous medium coincides with the flow velocity $v_{i}=v_{i}$, the force (17) is as follows: $f_{j}=e_{j m n} \rho v_{i} v_{m} \beta e_{n k i} \partial p / \partial X_{k}$. If to plug Levi-Civita contraction of a tensor $e_{n j m} e_{n k i}=\delta_{j k} \delta_{m i}-\delta_{j i} \delta_{m k}$ in this expression, we obtain the expression for the vortex force of the tensor field of interactions in the atmosphere in the form of:

$$
f_{j}=c^{-2} v_{m} v_{m} \frac{\partial p}{\partial X_{j}}-c^{-2} v_{j} v_{m} \frac{\partial p}{\partial X_{m}}
$$

Let's study this expression. We consider the one-dimensional case, when the velocity $v_{j}$ and the force $f_{j}$ lie along a straight line. Then expression (21) is identically zero.

We consider two-dimensional case of a plane vortex motion. Then the force (21) will be equal to: 


$$
f_{j}=c^{-2} v^{2} \partial p / \partial X_{j}
$$

This follows from the fact that the second summand in (21) is identically zero, since the velocity and pressure gradient are perpendicular to each other in case of circular motion.

Unlike the usual force $-\partial p / \partial X_{m}$ acting on unit volume, which balances the spatial fluctuations of pressure in the atmosphere, the force (22) is directed towards pressure increase. Most likely, this force is responsible for the appearance of vortex motion in the atmosphere in the form of cyclones and anticyclones. Since after the appearance of fluctuations in the pressure in the atmosphere, the force (22) increases this fluctuation, if vortex motion appears. In cyclones, the force (22) is directed from the center to the edges, and in anticyclones this force is directed towards the center. Thus, there is an inhomogeneous distribution of pressure in the atmosphere in the form of circular isobars in cyclones and anticyclones.

The force (22) is an additional force that appears in the swirling motion. In case of horizontal stationary rectilinear motion, the pressure gradient $-\partial p / \partial X_{m}$ is compensated by force $-\rho v_{i} \partial v_{i} / \partial X_{m}$, since the air pressure is the density of air energy, and it decreases in case of increase in the kinetic energy of the air currents (Bernoulli law). Therefore, the appearance of additional force (22), in case of the horizontal movement of air, results in redistribution of atmospheric pressure (air energy density), and vortex motion of air currents in the atmosphere.

Let's consider the three-dimensional case of air movement using the example of spouts and tornadoes. As is known, a tornado is a vortex movement of air in a horizontal plane in a column of air, which has the form of a funnel, with an expansion upwards. We calculate the vertical component of the vortex force $f_{3}$. According to (21), the component $f_{3}$ has the form:

$$
f_{3}=c^{-2} v_{\perp}^{2} \partial p / \partial X_{3}
$$

where $v_{\perp}^{2}=v_{1}^{2}+v_{2}^{2}$. Since in the horizontal plane: $v_{1} \partial p / \partial X_{1}+v_{2} \partial p / \partial X_{2}=0$.

Expression (23) is the lifting force acting in the tornado, since the gradient $\partial p / \partial X_{3}$ is directed upwards due to the upward expansion angle of the tornado funnel. It is clear from (23), that the higher the rotation speed in a tornado, the greater the lifting force.

It is known that the pressure in a tornado falls to half of the atmospheric pressure. A simple calculation shows that the force (23) can be greater than the gravity force, for example, at a tornado height of one kilometer and a horizontal speed of $100 \mathrm{~m} / \mathrm{s}$ :

$f_{3}=\beta \rho v_{\perp}^{2} \partial p / \partial X_{3}=\rho \times 2 \times 10^{-5} \times 10^{4} \times 0.5 \times 10^{5} \times 10^{-3}=\rho \times 10 \mathrm{~m} / \mathrm{c}^{2}$.

Here it was assumed that the pressure along the vertical changes uniformly, and the compressibility $\beta$ is inversely proportional to the pressure. These parameters are conditional, for example, the velocity can be smaller, and the pressure gradient may be larger. An important point is that the acceleration of free fall is really achievable for a lifting force in a tornado. 
If we ignore the gravity and the Peach-Koehler vertical force, the air movement along the funnel is possible in any direction, both upwards and downwards. This movement is analogous to the movement of water in the riverbed in case of narrowing or widening of the channel. For a stationary one-dimensional motion, the forces $-\partial p / \partial X_{3}$ and $-\rho v_{3} \partial v_{3} / \partial X_{3}$ balance each other-they are equal and opposite-directed.

If we take into account the vortex motion and the gravity force, then in case of increase in the rotation speed, the Peach-Koehler force can compensate for the gravity force. In addition, another force $-\rho v_{\perp} \partial v_{\perp} / \partial X_{3}$ is added, which is directed upwards, so in the narrower place of the tornado the speed of rotation is greater, which eventually results in greater lifting force in spouts and tornadoes.

A expectable question arises: how does the vortex force (21) relate to Euler vortex force? Since in the course of description of the vortex motion in the air the Euler vortex force is followed. It is obvious that the viscosity and additional members of the Navier-Stokes equation are not relevant to air currents in the form of cyclones and anticyclones. The vortex force of Euler hydrodynamics is as follows:

$$
f_{j}=\rho e_{j m n} v_{m} e_{n p q} \partial v_{q} / \partial X_{p}
$$

Let's take the convolution of the Levi-Civita tensors and write it in the form of:

$$
f_{j}=\rho v_{m} \frac{\partial v_{m}}{\partial X_{j}}-\rho v_{m} \frac{\partial v_{j}}{\partial X_{m}}
$$

In order to connect the vortex force $(15,17)$ with the Euler vortex force $(24)$, we note that it follows from the gauge condition (19), which has the form of a continuity equation, that the tensor interaction field can be written in the form: $A_{i j}=-c^{-2} v_{i} v_{j}$. In a continuous elastic medium, when $v_{j}=v_{j}$, the interaction tensor has the form of: $A_{i j}=-c^{-2} v_{i} v_{j}$. If we plug $A_{i j}=-c^{-2} v_{i} v_{j}$ in (1.17), we obtain a vortex force in the form of: $f_{j}=\rho c^{-2} v_{i} v_{m}\left(\frac{\partial\left(v_{i} v_{m}\right)}{\partial X_{j}}-\frac{\partial\left(v_{i} v_{j}\right)}{\partial X_{m}}\right)$ or after differentiation:

$$
f_{j}=\rho c^{-2} v^{2} v_{m}\left(\frac{\partial v_{m}}{\partial X_{j}}-\frac{\partial v_{j}}{\partial X_{m}}\right)+\rho c^{-2} v_{i} v_{m}\left(v_{m} \frac{\partial v_{i}}{\partial X_{j}}-v_{j} \frac{\partial v_{i}}{\partial X_{m}}\right)
$$

The first summand of the vortex force in (25) corresponds to the vortex member of the Euler hydrodynamics (24) multiplied by the dimensionless factor $c^{-2} v^{2}$. The second summand is additional, it is not taken into account in the Euler vortex force.

Moreover, in a gas, as a result of convolution: $A_{i j}=-\beta \delta_{i j} p$, the vortex force in the atmosphere has the form of (21). Therefore, the vortex force (17) in air is proportional to the pressure gradient, which explains the vortex movements of air in the atmosphere in the horizontal plane, connected with the change in pressure in the atmosphere (22). 
The relative multiplier $c^{-2} v^{2}$ appeared in (25) before the Euler vortex force in connection with the construction of the invariant Lagrangian (5) in field theory. The speed of sound is also clearly present in the gauge condition (19), from where the wave equation for mechanical oscillations in a continuous medium with the speed of sound follows. As is well known, the Maxwell's equations are also relativistic and contain the speed of light. The Kadić-Edelen equations were constructed from similar considerations and contain the speed of sound (5 - 7). Taking into account that mechanical oscillations actually propagate with the speed of sound (19), there is no reason to be doubtful of the correctness of the theory construction.

The relative multiplier $c^{-2} v^{2}$ is absent in the Euler equations, since they are obtained by differentiation if the composite function by parts. According to (25), the vortex member of the Euler force gives a correct force only if a velocity of a continuous medium is equal to the speed of sound. For example, if speeds are lower than speed of sound by one order of magnitude, it gives a force that exceeds the real force by two orders of magnitude, and at speeds greater than 1 $\mathrm{km} / \mathrm{s}$, it gives a force one order less than real one.

Thus, the turbulences in gas and atmosphere are described by the force (21), and not by the vortex member in the Euler Equation (24), since the vortex member in the Euler equations does not take into account the relative velocity of the continuous medium in comparison with the speed of sound. This result is nontrivial, it is connected with the minimal interaction and the Lagrangian $(2,5$, 19). In principle, the Euler equations cannot describe turbulence, since the vortex member in the Euler equation does not take into account the difference between the speed of sound and the velocity of a continuous elastic medium (25). It is known that turbulence increases with increasing speed and manifests itself at speeds commensurate with the speed of sound, which corresponds to the expression (21).

In fact, forces $(16,17)$ in a continuous medium generalize the potential and Euler vortex forces, respectively.

In case of substitution $A_{i j}=-\beta \delta_{i j} p$, the centrally symmetric force (16) is as follows:

$$
f_{j}=-p_{i} \partial v_{i} / \partial X_{j}-p_{j} \beta \partial p / \partial T
$$

The first summand in (26) corresponds to the potential member of the Euler equations. The second summand was not taken into account earlier when describing motions in a continuous medium.

Let's suppose that the pressure in the atmosphere changes uniformly. Then (26) has the form: $\partial p_{j} / \partial T=-p_{j} \beta \partial p / \partial T$. This equation is easily integrated and its solution has the form: $p_{j}=p_{j 0} \exp \left(\beta p_{0}-\beta p\right)$, where $p_{0}$ is the initial pressure, and $p_{j 0}$ is the initial momentum.

Whence it follows that in the homogeneous case, if the pressure in the atmosphere decreases, the wind will increase, and if the pressure increases, then wind will cease, and everyone knows this. 
It should be also noted that the second summand in (26) plays a very important role in the propagation of sound waves, since it annihilates the force (26) for the sound wave solutions. The potential member [18], which is responsible for the kinetic energy of the continuous medium, is neglected in the description of sound in Euler equations. It is obvious that it cannot be neglected, since the sound waves themselves are associated with the motion of a continuous medium. The inclusion of the second summand in (26) solves this problem.

Consequently, when describing motions in a continuous medium, it is necessary to use not the Euler equations, but the forces $(16,17)$ obtained from the minimum of the action for the momentum in the tensor interaction field (10), which in the gas or liquid have the form of $(21,26)$.

\section{Explanation of High-Temperature Plasma by the Action of the Centrally Symmetric Force of the Tensor Interaction Field in a Gas}

Let's consider the simplest case how the homogeneous constant centrally symmetric force $f_{j}=p_{i} \varepsilon_{i j}$ (16) manifests itself in a gas.

In a gas, the intensity $\varepsilon_{i j}$ is given by the scalar value: $\varepsilon_{i j}=\delta_{i j} \varepsilon$, just as the strain tensor in the gas is given by the scalar pressure $\sigma_{i j}=-\delta_{i j} p$. Let's write the equation of motion for a gas molecule in a certain volume, where a centrally symmetric intensity $\varepsilon_{i j}=\delta_{i j} \varepsilon$ acts on the momentum of the gas molecules. According to (16), this equation of motion is as follows:

$$
\partial p_{j} / \partial t=p_{j} \varepsilon
$$

As is known, the solution of Equation (26) is the exponential:

$$
p_{j}=p_{j 0} \exp (\varepsilon \tau)
$$

where $\tau$ is the time during which the field acts on gas molecules. According to this solution, in the volume where the field acts, all gas molecules will be accelerated exponentially. In this case, the scalar field $\varepsilon$ will increase only the momentum of all gas molecules, without changing the direction of their motion during a free path. Consequently, the field $\varepsilon$ in a certain volume of the gas increases the kinetic energy of the gas molecules.

If the field $\varepsilon$ is large, then its action in a certain volume of gas will result in sharp increase in the kinetic energy of all gas molecules in this volume. It is known that the average kinetic energy of gas molecules determines its temperature. Consequently, the action of a large field $\varepsilon$ will lead to the appearance of a high-temperature plasma in a gas. Since in case of the exponential growth of the kinetic energy of molecules, a moment will surely arise when the collisions of the molecules cease to be elastic and electromagnetic radiation will appear.

The action of a large field $\varepsilon$ in a certain volume results in high-temperature plasma and describes an explosion. It is obvious that in case of explosion the temperature or average kinetic energy of the gas molecules in a certain volume sharply increases. As a result of a sharp increase in temperature, the gas pressure in such volume also sharply increases, and therefore a shock wave is formed. 
It is believed that the explosion is associated with a chemical or nuclear reaction. It should be noted that this does not contradict the conclusion just made, because the heat released during the reaction is the change in the average kinetic energy of the gas molecules.

However, there are many phenomena of nature where there is no chemical or nuclear reaction, but there is an explosion and a high-temperature plasma. For example, an explosion is observed in case of shot of railgun, although it is known that there is no gunpowder in the gun [20].

The same phenomenon is observed when meteorites burn in the Earth's atmosphere, when chondrites (stones consisting mainly of iron and silicon) burn and vaporize in high-temperature plasma in the snap of a finger. There are attempts to justify such phenomena by friction in the atmosphere, density of which is a million times smaller than on Earth.

The explosion of the Chelyabinsk meteorite is another example of an explosion without a chemical or nuclear reaction. In the explosion of the Chelyabinsk meteorite, a gas (air) also exploded as a result of the centrally symmetric force of the tensor interaction field acting on the momentum of air molecules (16). At first, the meteorite broke into flames when the air, as a continuous medium or "dense layers of the atmosphere", was destroyed at an altitude of $95-100 \mathrm{~km}$, and a shell of high-temperature plasma formed around it. At a point about thirty kilometers above the Earth, the pressure in high-temperature plasma became about 2 - 3 thousand atmospheres due to the natural increase in atmospheric density during the fall of the meteorite. This pressure crushed the meteorite (chondrite) into molecules and turned it into a "stone cloud", consisting mainly of molecules of compounds of iron and silicon.

Then the "stone cloud" exploded like 500 thousand tons of trotyl with a pressure of billions of atmospheres in the sky above Chelyabinsk, while the weight of the meteorite (chondrite) itself was only 10 thousand tons. It was not trotyl, but the usual chondrite stone which in terms of its composition is similar to ore. Ore melts in blast furnaces, but never explodes. The increase in pressure during the formation of a "stone cloud" to a billion atmospheres can be formally explained by the increase in pressure in the atmosphere due to an increase in its density from the air density at an altitude of $30 \mathrm{~km}$ to the density of the "stone cloud". In fact, the process of change in the pressure during an explosion is more complex, but a detailed description of the explosion is beyond the scope of this paper.

Now it becomes clear that without centrally symmetric strain $\varepsilon_{i j}=\delta_{i j} \varepsilon$ it is impossible to explain and describe a high-temperature plasma. Since sharp increase in the temperature of the gas in case of an explosion in a certain volume is just described by an exponential increase in the kinetic energy of gas molecules in this volume. Such a description of a high-temperature plasma is obvious. Just earlier, the reason for the appearance of the field $\varepsilon(6,7)$ was not clear, and it was unclear how it works (27). Therefore, until now the explosions are measured by the TNT equivalent, and not by the power characteristic-by the centrally symmetric intensity of the interaction tensor (8). Most likely, all explosions are 
similar, and the mechanism of acceleration of gas molecules exponentially (27) is the same during explosions.

The Equation (27) has solutions in the form of an exponential only because the charge for the intensity $\varepsilon$ is momentum (16). The fact that the momentum $p_{i}=\hbar \kappa_{i}$ is a charge was proved above by direct calculations of the extended derivative (2) induced by the translation subgroup.

How can electromagnetic phenomena be explained without knowing the existence of electrical and magnetic intensity? Without the equations of state for the electromagnetic field (without Maxwell equations), there would be no capacitors, no transformers, no cell phones, but there would be knowledge of electromagnetic forces at the level of the verification of the attraction of electrified or magnetized items.

It is also impossible to describe the phenomena of nature without the equations of state for the intensities of the 4-tensor interaction $(6,7)$. It is impossible to replace intensity $(1,8)$ and forces $(16,17)$ by any other forces in nature.

We note that the writing of the action (10) became possible only after the derivation of the fundamental minimal interaction with the compensating tensor field $A_{i j}$ (2), the charge of which is a quantum momentum $p_{i}=\hbar \kappa_{i}$, which ultimately defines this interaction.

The connection between the theory of Landau phase transitions for OP with local translational properties [2] and quantum mechanics is not unexpected, since the localization of the transformation properties of the OP in the Landau theory $k_{i}=k_{i}\left(X_{j}\right)$, which was used in the minimal interaction construction (2, $3)$, means that in a macroscopic small volume [10] with the coordinate $\boldsymbol{X}$ the physical state is described by the wave function. In other words, it is possible to determine locally the wave vector in a macroscopic small volume with a coordinate $\boldsymbol{X}$. But this is the basic postulate of quantum mechanics, which says that any state can be locally represented as a wave.

\section{Conclusions}

Consequently, we have shown that the tensor interaction field: $v_{i}, A_{i j}$, like the electromagnetic field, has two intensities-vortex $\rho_{i j} \quad(1)$ and centrally symmetric $\varepsilon_{i j}(8)$, which act on the momentum as on charge $(16,17)$ and are determined from the equations of state $(6,7)$, analogous to the Maxwell equations.

The vortex force (17) in a solid is the Peach-Koehler force (18). In the general case, it acts on the momentum flux (17), just as the Lorentz force acts on the flow of charged particles.

The centrally symmetric force (16) acts on the momentum in the same way as the Coulomb force acts on the electric charge. The action of a large centrally symmetric intensity in a certain volume on the gas molecules: $\partial p_{j} / \partial t=p_{j} \varepsilon$ (27) results in an exponential increase in the magnitude of the momentum $p_{j}=p_{j 0} \exp (\varepsilon t)$, and consequently, of the kinetic energy of the gas molecules in this volume, which explains and describes the high-temperature plasma. Here 
it is assumed that the field $\varepsilon$ I not changed significantly during the mean free time.

The action of a large vortex force $f_{j}=e_{j m n} p_{i} v_{m} \rho_{i n}$ (17) is analogous to the action of a large Lorentz force in a tokamak. A large vortex force in space captures momentums. This explains the phenomenon of "black holes", an area with a large gravitational field in the space. A source of high intensity $\rho_{i j}$ (1) in a gas is a large pressure in a certain volume (7). Consequently, a large density is observed in this volume, with a large gravitational field. But the gravitational field does not act on photons, since photons do not have mass. However, a large vortex force (17) acts on the photons, since photons have momentum, so they also do not return from the "black holes".

The vortex force of the tensor interaction field in a continuous medium generalizes the Euler vortex force in a continuous medium. In the case of horizontal vortex motion in the atmosphere, the vortex force $(21)$ is proportional to the pressure gradient and is directed towards increase in the pressure $f_{j}=c^{-2} v^{2} \partial p / \partial X_{j}$ (22), rather than decrease in the pressure, as the force $f_{j}=-\partial p / \partial X_{j}$ acts in the atmosphere. Therefore, the vortex force (22) is responsible for the appearance of stable regions of low and high pressure in the atmosphere, in cyclones and anticyclones.

An expectable question arises when forces act $(16,17)$ and when quantum effects are observed, for example in a gas? since the gas is not always a high-temperature plasma. What are the conditions for an explosion and what is an explosion?

What is the vortex intensity $\rho_{i j}$ in the air?

We will answer all these questions in the next paper. It will be shown that the analogy between the compensating fields: the interaction tensor $A_{i j}$ and the electromagnetic potential $A_{j}$ can be traced not only for the forces $(16,17)$ and the equations of state $(6,7)$, but also for phase transformation or phase transitions of these fields.

In 1964, Higgs described the phase transitions when the unobservable compensating fields of minimal interaction, for example $(2,3)$, change their symmetry and materialize [21] [22]. He demonstrated that a spontaneous symmetry breaking of the compensating fields is possible, when an unobservable compensating field becomes observable.

For an electromagnetic field, the Higgs transition is a transition to the superconducting state with the Meissner effect [22]. Such transitions are well studied. In the superconducting state, the electromagnetic potential materializes; it becomes proportional to the current density, and the London equations [12] [22] $A_{i}=-\delta^{2} j_{i}$ are performed, where $j_{i}$ is the current density, and $\delta$ is the penetration depth of the magnetic field into a superconductor. In this case, the gradient symmetry of the Maxwell equations is violated.

In the next paper it will be demonstrated that for a compensating tensor field of interaction $A_{i j}(2)$, a phase transition with a violation of the gradient symmetry of the state Equations (6), (7) is a transition to the elastic state of a conti- 
nuous medium. In an isotropic continuous medium with $\rho$ density, the tensor $A_{i j}$ materializes and becomes proportional to the strain tensor: $\sigma_{i j}=c^{2} \rho A_{i j}$. This correlation is similar to the London equation in superconductivity.

Just like that the superconducting state holds the magnetic field, the continuous medium holds the field $\rho_{i j}$ (1), which is realized as linear defects (or "holes"), because the continuous medium "does not tolerate emptiness". The destruction of a continuous elastic medium in a solid is always accompanied by the appearance of dislocations and cracks. Such processes are called plastic deformations and are just described by the distortion tensor $A_{i j}$ (1).

The next paper will demonstrate that the destruction of a continuous elastic medium is a phase transition of the destruction of the material or elastic state of the tensor field $A_{i j}$. This phase transition is analogous to the phase transition of the destruction of the superconducting state of the electromagnetic field. Just as a critical current destroys the superconducting state, the critical pressure in the same manner results in penetration of the field $\rho_{i j}$ into the continuous medium, resulting in the destruction of continuous elastic medium. In a solid, the cracks form as a result of such a transition, and lightning and explosions occur in the gas.

\section{Conflicts of Interest}

The author declares no conflicts of interest regarding the publication of this paper.

\section{References}

[1] Landau, L.D. and Lifshitz, E.M. (1987) Field Theory. 2nd Edition, Nauka, Moscow.

[2] Braginsky, A.Y. (2014) Vortex State (A Phase) of $\mathrm{Fe}_{0.5} \mathrm{Co}_{0.5} \mathrm{Si}, \mathrm{MnSi}$, and FeGe Helimagnets as a Continuum Dislocation Density Distribution in a Magnetic Sublattice. Bulletin of the Russian Academy of Sciences: Physics, 78, 754-759.

[3] Landau, L.D. and Lifshitz, E.M. (1987) Elasticity Theory. 7th Edition, Nauka, Moscow.

[4] Mühlbauer, S., Binz, B., Jonietz, F., et al. (2009) Skyrmion Lattice in a Chiral Magnet. Science, 323, 915-919. https://doi.org/10.1126/science.1166767

[5] Yu, X.Z., Onose, Y., Kanazawa, N., et al. (2010) Real-Space Observation of a Two-Dimensional Skyrmion Crystal. Nature, 465, 901-904.

https://doi.org/10.1038/nature09124

[6] Kanazawa, N., Onose, Y., Yu, X.Z., Kimoto, K., et al. (2011) Near Room-Temperature Formation of a Skyrmion Crystal in Thin-Films of the Helimagnet FeGe. Nature Materials, 10, 106-109.

[7] De Gennes, P.G. (1972) An Analogy between Superconductors and Smectics A. Solid State Communications, 10, 753-756. https://doi.org/10.1016/0038-1098(72)90186-X

[8] Braginsky, A.Y. (2003) Distorted States of Smectic A in the Vicinity of the Nematic-Smectic-A Phase Transition. Physical Review, 67, 174113-174119. https://doi.org/10.1103/PhysRevB.67.174113

[9] Slavnov, A.A. and Fadeev, L.D. (1990) Gauge Fields: Introduction to Quantum 
Theory. Addison-Wesley, Redwood City, CA.

[10] Lifshitz, E.M. (1941) On the theory of Phase Transitions of the Second Kind. $Z h$. Exp. Teor. Fiz, 11, 255-281.

[11] Braginsky, A.Y. (1990) Phenomenological Theory of Phase Transitions to States with Local Symmetry. Solid State Physics, 32, 2121-2125.

[12] Lifshits, E.M. and Pitaevskiy, L.P. (1980) Course of Theoretical Physics, Vol. 9: Statistical Physics: Pt. 2: Theory of the Condensed State. Pergamon, New York.

[13] Kadić, A. and Edelen, D.G.B. (1983) A Gauge Theory of Dislocations and Disclinations. In: Lecture Notes in Physics, Vol. 174, Heidelberg, Springer, 168. https://doi.org/10.1007/3-540-11977-9

[14] Lazar, M. (2011) On the Fundamentals of the Three-Dimensional Translation Gauge Theory of Dislocations. Mathematics and Mechanics of Solids, 16, 253-264. https://doi.org/10.1177/1081286510370889

[15] De Wit, R. (1960) The Continuum Theory of Stationary Dislocations. Solid State Physics, 10, 249-292.

[16] Braginsky, A.Y. (2007) Inhomogeneous Ordered States and the Translational Nature of the Gauge Group in the Landau Continuum Theory: II. Applications of the General Theory. Zh. Exp. Teor. Fiz, 132, 45-50.

[17] Musienko, A.I. and Manevich, L.I. (2004) Classical Mechanical Analogs of Relativistic Effects. Physics-Uspekhi, 47, 797-820. https://doi.org/10.1070/PU2004v047n08ABEH001402

[18] Landau, L.D. and Lifshitz, E.M. (1988) Hydrodynamics. 6th Edition, Nauka, Moscow.

[19] Peach, M.O. and Koehler, J.S. (1950) The Forces Exerted on Dislocations and the Stress Fields Produced by Them. Physical Review, 80, 436-439. https://doi.org/10.1103/PhysRev.80.436

[20] https://www.youtube.com/watch?time_continue=3\&v=zdkn-OG9kI0

[21] Itzkson, K. and Zuber, J.B. (1980) Quantum Field Theory. McGraw-Hill, New York.

[22] Higgs, P.W. (1964) Broken Symmetries and the Masses of Gauge Bosons. Physical Review Letters, 13, 508-509. https://doi.org/10.1103/PhysRevLett.13.508 